\title{
The spatial analysis of landscape ecological stability and ecological security in the steppe regions of Russia
}

\author{
Alexander A. Chibilyov (jr.) ${ }^{1}$, Dmitry S. Meleshkin¹, Dmitry V. Grigorevsky¹ \\ 1 Orenburg Federal Research Center, Institute of Steppe of the Ural Branch of the Russian Academy of \\ Sciences, Orenburg, 460000, Russia \\ Corresponding author: Dmitry V. Grigorevsky (grag92@mail.ru)
}

Academiceditor:R. Yakovlev | Received 25 November 2021 | Accepted 9December 2021 | Published23 December 2021

http://zoobank.org/39BE7CF2-2A97-486B-8F5B-8A70AFD5F59C

Citation: Chibilyov (jr.) AA, Meleshkin DS, Grigorevsky DV (2021) The spatial analysis of landscape ecological stability and ecological security in the steppe regions of Russia. Acta Biologica Sibirica 7: 593-607. https://doi. org/10.3897/abs.7.e78450

\begin{abstract}
The ecological-economic security [EES] and the landscape-ecological stability of the steppe regions of Russia are the main factors of national security and indicators of the sustainability of socio-economic development. Therefore, it is advisable to consider them based on large-scale studies. The study territory is a mesoregion, including the steppe zone of Russia. A comprehensive analysis of the economic development, social sector, and ecological condition of the territory became the basis for assessing the EES of the regions. We assessed landscape-ecological stability by calculating the coefficient of ecological stability of the landscape. Based on reliable information, we formed a database that comprises 37 indicators for 18 steppe regions of Russia. Using the coefficients and integral indices, we compiled an integrated schematic map. As a result, we established that the level of stability of the ecological landscape increases to the northeast of the studied territory. We observed the maximum value of the ecological and economic security index (1.8) in Krasnodar Krai, the Republic of Bashkortostan, and Novosibirsk Krai. Furthermore, we characterized the regions where the coefficient of ecological landscape stability exceeded 1 by indicators of ecological and economic security, presented in the ratio 1/1.5/2.
\end{abstract}

\section{Keywords}

Ecological-economic security, landscape-ecological stability, economic development, social sector, ecological condition, steppe zone, Russia

Copyright Alexander A. Chibilyov (jr.) et al. This is an open access article distributed under the terms of the Creative Commons Attribution License (CC BY 4.0), which permits unrestricted use, distribution, and reproduction in any medium, provided the original author and source are credited. 


\section{Introduction}

The growth of economic activity has had a negative impact on the steppe zone of Russia. It is expressed in (1) environmental pollution, (2) reduction in species biodiversity, (3) depletion of natural resources, (4) degradation of landscapes, and (5) the gap between natural relationships. In light of recent events related to problems of stable socioeconomic development and the coronavirus pandemic, the most significant tasks in Russia (current and future) are to (1) solve the problem of protection of nature, (2) improve the sanitary and epidemiological situation in urbanized areas, and (3) raise the standard of living of the population.

In general, the territory of the steppe regions of Russia is marked by an unfavorable environmental situation. Territorial differences in indicators that characterize the situation can be revealed in the cartographic analysis of the landscape and ecological stability and ecological-economic security [EES] of the steppe regions of Russia.

\section{Material and methods}

Based on the methods of (Klementova and Heinige 1995), (Baranov 1995; 2001; 2012), (Kochurov 2005), and (Klyushin and Shormanov 2015), we assessed landscape-ecological stability by calculating the coefficient of ecological stability of the landscape [CELS]. These authors describe a methodological approach to developing a system for assessing the landscape and environmental sustainability of the territory for the formation of ecologically balanced landscapes. Foreign scientists proposed a method for assessing agricultural landscapes (Stinner et al. 1989; McNeely and Scherr 2003; Malezieux 2012). Furthermore, we compared territories with stable landscape components (hayfields, pastures, perennial plantings, forests, deposits, swamps, strictly protected natural areas) and unstable ones (arable land, plots for construction, roads, damaged land, reclaimed land). This method allowed us to assess the ecological and landscape stability of the area, taking into account the qualitative and quantitative parameters of the biotic and abiotic components in the land fund of the regions.

This method is based on the comparison of the areas occupied by stable and unstable components of landscapes. The coefficient contributes to assessing landscape-ecological stability, which combines the qualitative and quantitative parameters of biotic and abiotic elements in the natural-territorial complex. According to the methodology, the territory is divided into two groups of landscapes (stable and unstable), the proportion of which was calculated using the following formula:

$$
\text { CELS } \frac{\sum F_{\text {st }}}{\sum F_{\text {unst }}},
$$


where:

$\mathrm{F}_{\text {st }}$ - areas marked with stable landscape components;

$\mathrm{F}_{\text {unst }}$ - areas characterized by unstable components.

Stable and unstable landscape components are buffering; they partially compensate for themselves. Assessment of landscape and ecological stability at the regional level is limited by a coefficient (Saprin 2017). The lack of information about areas with water and swamps in our study can be explained by the limited data used to record materials in open statistical collections. We assessed CELS using the scale represented in Table 1.

Table 1. A CELS rating scale

\begin{tabular}{ll}
\hline CELS & Description of the ecological stability of the landscape \\
\hline$\leq 0.5$ & Sufficiently unstable \\
$0.5-1.0$ & Unstable \\
$1.0-3.0$ & Conditionally stable \\
$3.0-4.5$ & Sufficiently stable \\
$\geq 4.5$ & Stable \\
\hline
\end{tabular}

According to the method, the stable components of the landscape $\left(\mathrm{F}_{\mathrm{st}}\right)$ are (1) hayfields $\left(\mathrm{y}_{1}\right),(2)$ pasture $\left(\mathrm{y}_{2}\right),(3)$ perennial plantings $\left(\mathrm{y}_{3}\right),(4)$ forests $\left(\mathrm{y}_{4}\right),(5)$ deposits $\left(\mathrm{y}_{5}\right),(6)$ swamps $\left(\mathrm{y}_{6}\right)$, and (7) strictly protected natural areas $\left(\mathrm{y}_{7}\right)$. Unstable components $\left(\mathrm{F}_{\text {unst }}\right)$ are $(1)$ arable land $\left(\mathrm{y}_{8}\right),(2)$ plots under construction $\left(\mathrm{y}_{9}\right),(3)$ a network of streets (streets, driveways, embankments, etc.) $\left(\mathrm{y}_{10}\right),(4)$ damaged land with some indicators (constructions, buildings, paths, lawns, and ornamental plantations), $\left(\mathrm{y}_{11}\right)$, reclaimed land $\left(\mathrm{y}_{12}\right)$ (Table 2$)$.

Two concepts contribute to assessing the level of ecological security: anthropogenic (solving environmental problems) and biospheric (identifying the zone of ecosystem stability). The anthropogenic concept was considered in the works of Vlasova (2008); (Holling 1978); and (Chapin et al. 2010). Methods and criteria for ecological security within the framework of the biospheric concept were developed by (Vlasova 2008); (Volovich 2012); (Rusak 2011); (Tronin 2013); (Carpenter et al. 1999); (Jongman et al. 2004); and (Zhaoxue and Linyu 2010). The combined analysis of the level of economic development, social sector, and ecological condition of the territory is the main component of assessing the EES of the steppe regions of Russia. We used and updated the methodology of (Glinsky et al. 2015). Using current and reliable data published in the public domain, we formed a database on EES indicators of the studied regions. Later, these indicators were combined into three groups.

Usually, these sources present data in absolute terms ( $\mathrm{t}$, ha, unit, $\mathrm{km}^{2}$ ), which are characterized by various impacts. Taking into account the EES goal of the analysis 
of EES and the improvement of the objectivity of the research, we calculated some indicators corresponding to the regions population size in the studied regions (Table 2).

Group 1. The economic development index $\left(\mathrm{P}_{\mathrm{I}}\right)$ (parameters that adversely affect the integrated indicators are marked with asterisk $\left.{ }^{*}\right): \mathrm{x}_{1}-$ the volume of goods shipped by production; works and services sold by their own force, million rubles/ person; $\mathrm{x}_{2}$ - turnover of retail trade per capita, thousand rubles; $\mathrm{x}_{3}$ - volume of paid services per capita, thousand rubles; $\mathrm{x}_{4}$ - investment in fixed assets per capita, thousand rubles; $\mathrm{x}_{5}$ - the share of unprofitable organizations ${ }^{*}, \%$; $\mathrm{x}_{6}$ - the volume of agricultural production per capita, thousand rubles; $\mathrm{x}_{7}$ - commissioning of residential buildings per 1,000 people ( $\mathrm{m}^{2}$ of the total area of residential buildings).

Group 2. Index of social development $\left(\mathrm{P}_{\mathrm{II}}\right): \mathrm{x}_{8}$ - total birth rate; $\mathrm{x}_{9}$ - total death rate $^{*} ; x_{10}$ - infant mortality rate $x_{11}$ - migratory growth rate; $x_{12}$ - share of preschool children per 1,000 people; $\mathrm{x}_{13}$ - share of students enrolled in educational programs of junior, senior and general secondary education per 1,000 people; $\mathrm{x}_{14}$ - morbidity per 1,000 people ${ }^{*} x_{15}$ - share of inpatient accommodation per 10,000 people; $\mathrm{x}_{16}$ - share of doctors per 10,000 people; $\mathrm{x}_{17}$ - share of unemployed registered in state employment agencies per 1,000 people ${ }^{\star} ; x_{18}$ - share of crimes registered per 1,000 people*.

Group 3. Index of the ecological condition of the area $\left(\mathrm{P}_{\mathrm{III}}\right): \mathrm{x}_{19}$ - the amount of pollutants released into the atmosphere per 1,000 people $\mathrm{x}_{20}$ - expenses for the protection of nature, including payment for environmental services per capita; $\mathrm{x}_{21}$ the share of arable land under crops from the total area*; $x_{22}$ - the share of land with settlements*; $x_{23}$ - the share of land used in industry and energy sectors, as well as for transport, communication, broadcasting, television, computer science, defense, security, and other purposes ${ }^{\star} ; \mathrm{x}_{24}$ - the percentage of forest land in the territory; $\mathrm{x}_{25}$ - the share of capture, depolarization and neutralization of pollutants from stationary sources of pollution.

The selected indicators are characterized by heterogeneity, disparity, and diversity of their impact. To eliminate these defects, we normalized the design parameters:

$$
\begin{aligned}
x_{i j}^{\text {norm }} & =\frac{x_{i j}-\min x_{i j} j}{\max x_{i j} j-\min x_{i j} j}, \\
x_{i j}^{n o r m} & =\frac{\max x_{i j} j-x_{i j}}{\max x_{i j} j-\min x_{i j} j},
\end{aligned}
$$


where:

$x_{i j}^{\text {norm }}$ - the normalized value of $\mathrm{j}$-indicator that $\mathrm{i}$-municipality has;

$\max x_{i j} j$ - the maximum value of $\mathrm{j}$-indicator in the studied municipalities;

$\min x_{i j} j$ - a minimal value of $j$-indicator in the examined municipal units;

$i$ - the number of the municipality;

$j$ - the number of the indicator.

If the integral assessment parameter has a positive effect, the normalization is calculated using the formula (2). If it has a negative effect, the normalization is calculated using the formula (3).

In the future, the multidimensional averages calculated for each group contribute to the transition from multidimensional indicators to one-dimensional parameters (4).

$$
\overline{P_{m i}}=\frac{1}{k} \sum_{j=1}^{k} x_{i j}^{\text {norm }} \text {, }
$$

where:

$\overline{P_{m i}}$

$m i$ - the average indicator of a specific group of indicators of EES;

$k$ - the indicators in the group.

The integral indicator of ecological and economic security (5) is calculated by the sum of the multidimensional average indicators in each group:

$$
I_{i}^{e e s}=\sum_{m=1}^{3} \overline{P_{m i}}
$$

where:

$I_{i}^{\text {ees }}-$ the integral indicator of EES;

$m-$ the number of groups.

To assess the spatial distribution of the level of ecological and economic security, we should identify regions where, as a result of normalization, a maximum of aggregated indicators can be identified, reduced to 1 (Table 3 ). 
Table 2. Initial indicators to calculate the EES CELS and the index of EES of the steppe regions of Russia

\begin{tabular}{|c|c|c|c|c|c|c|c|c|c|c|c|c|c|c|c|c|c|c|}
\hline 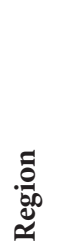 & 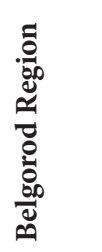 & 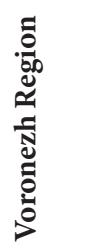 & 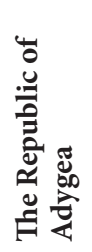 & 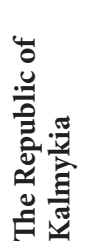 & 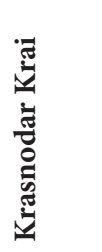 & 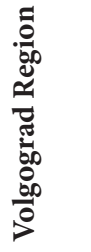 & 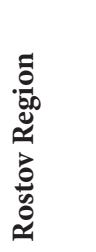 & 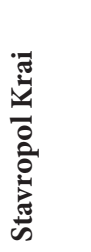 & 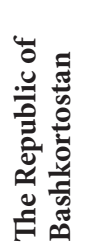 & 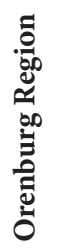 & 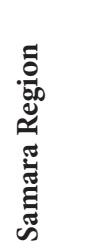 & 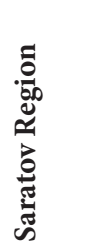 & 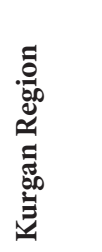 & 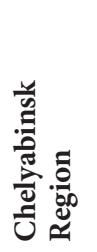 & 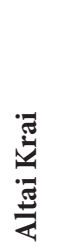 & 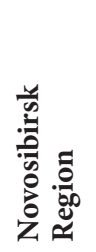 & 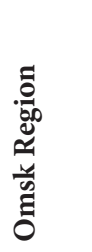 & 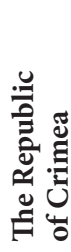 \\
\hline S & 27.1 & 52.2 & 7.8 & 74.7 & 75.5 & 112.9 & 101.0 & 66.2 & 142.9 & 123.7 & 53.6 & 101.2 & 71.5 & 88.5 & 168.0 & 177.8 & 141.1 & 26.1 \\
\hline $\mathrm{P}$ & 1547.7 & 2327.8 & 454.8 & 272.6 & 5648.2 & 2507.5 & 4202.3 & 2795.2 & 4051.0 & 1963 & 3183.0 & 2440.8 & 834.7 & 3475.7 & 2332 & 2793.4 & 1944.2 & 1911 \\
\hline GRP & 507.7 & 371.7 & 218.6 & 244.0 & 394.1 & 307.7 & 320.6 & 238.1 & 344.7 & 419.3 & 424.1 & 274.1 & 240.6 & 388.0 & 218.1 & 408.4 & 334.9 & 187.8 \\
\hline $\mathrm{y}_{1}$ & 55.8 & 159.0 & 4.9 & 103.2 & 63.1 & 206.9 & 88.4 & 104.9 & 1266.7 & 698.0 & 67.0 & 122.2 & 559.0 & 591.1 & 1235 & 2197.9 & 1096.2 & 1.9 \\
\hline $\mathrm{y}_{2}$ & 399.3 & 776.8 & 85.7 & 5363.6 & 531.1 & 2652.8 & 2459.2 & 1625.8 & 2346.1 & 3979 & 847.5 & 2400.5 & 1024.8 & 1352.0 & 2789 & 2315.0 & 1265.5 & 433.6 \\
\hline $\mathrm{y}_{3}$ & 34.0 & 52.8 & 9.3 & 2.5 & 125.2 & 42.8 & 58.2 & 44.2 & 43.6 & 23.0 & 42.3 & 39.9 & 12.4 & 38.3 & 27.8 & 33.6 & 26.5 & 75.8 \\
\hline $\mathrm{y}_{4}$ & 241.9 & 482.4 & 288.8 & 32.6 & 1541.3 & 591.0 & 293.0 & 110.2 & 5765.6 & 618.6 & 685.6 & 614.2 & 1759.5 & 2707.3 & 4029 & 4799.2 & 4667.7 & 266.2 \\
\hline $\mathrm{y}_{5}$ & N/A & 41.9 & 0.3 & 10.6 & 0.2 & 4.7 & N/A & 14.0 & 0.0 & N/A & 103.5 & 0.0 & 459.3 & 55.0 & 298.9 & 81.0 & 175.9 & 10.6 \\
\hline $\mathrm{y}_{6}$ & 22.5 & 40.6 & 4.0 & 123.5 & 179.6 & 35.2 & 55.0 & 28.8 & 50.8 & 15.3 & 42.0 & 19.2 & 383.9 & 192.7 & 374.7 & 3059.6 & 2026.8 & 5.2 \\
\hline $\mathrm{y}_{7}$ & 2.8 & 35.1 & 92.8 & 121.6 & 378.8 & 33.1 & 11.4 & 0.5 & 412.0 & 102.5 & 138.8 & 30.5 & 9.7 & 64.2 & 44.9 & 2.7 & 0.7 & 19.9 \\
\hline $\mathrm{y}_{8}$ & 1645.2 & 3046.2 & 259.6 & 836.9 & 3985.4 & 5854.0 & 5907.3 & 3998.6 & 3670.5 & 6115 & 2937.5 & 5981.1 & 2402.6 & 3058.8 & 6654 & 3772.1 & 4156.6 & 1271 \\
\hline $\mathrm{y}_{9}$ & 73.1 & 113.4 & 22.1 & 32.2 & 202.9 & 165.9 & 150.8 & 107.5 & 132.1 & 158.7 & 103.0 & 113.3 & 49.1 & 137.8 & 131.9 & 102.4 & 93.9 & 118.8 \\
\hline $\mathrm{y}_{10}$ & 57.9 & 121.1 & 18.8 & 65.1 & 196.0 & 117.6 & 220.5 & 147.9 & 260.1 & 184.7 & 123.7 & 149.4 & 86.3 & 145.5 & 195.5 & 166.8 & 150.7 & 43.4 \\
\hline $\mathrm{y}_{11}$ & 6.5 & 1.9 & 0.3 & 4.0 & 5.4 & 3.0 & 7.1 & 3.4 & 17.2 & 13.0 & 3.9 & 2.4 & 1. & 31.8 & 3.6 & 1.7 & 5.0 & 1.5 \\
\hline $\mathrm{y}_{12}$ & 42.2 & 78.8 & 27.3 & 48.3 & 426.0 & 180.7 & 259.6 & 241.2 & 73.2 & 63.4 & 140.6 & 257.8 & 30.6 & 69.5 & 72.9 & 83.7 & 124.5 & 397.3 \\
\hline $\mathrm{x}_{1}$ & 578.7 & 244.5 & 1316 & 13.3 & 217.9 & 412.7 & 269.7 & 144.2 & 423.1 & 495.1 & 512.0 & 227.3 & 160.5 & 500.2 & 165.3 & 251.8 & 529.6 & 79.2 \\
\hline $\mathrm{x}_{2}$ & 217.1 & 237.0 & 209.6 & 77.9 & 243.2 & 154.9 & 218.6 & 182.3 & 216.8 & 156.3 & 203.4 & 145.7 & 136.2 & 148.8 & 150.4 & 179.6 & 169.3 & 133.9 \\
\hline $\mathrm{x}_{3}$ & 53.8 & 55.4 & 29.7 & 21.4 & 92.8 & 55.5 & 54.8 & 56.9 & 64.4 & 45.1 & 54.9 & 40.0 & 37.2 & 47.1 & 40.4 & 57.6 & 51.1 & 45.2 \\
\hline $\mathrm{x}_{4}$ & 86.6 & 119.8 & 69.5 & 47.3 & 85.5 & 73.4 & 60.0 & 56.6 & 66.0 & 103.7 & 81.3 & 63.3 & 32.3 & 74.4 & 46.9 & 70.2 & 60.8 & 154.8 \\
\hline
\end{tabular}




\begin{tabular}{|c|c|c|c|c|c|c|c|c|c|c|c|c|c|c|c|c|c|c|}
\hline 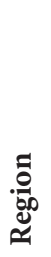 & 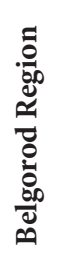 & 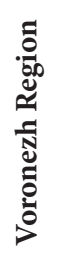 & 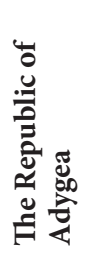 & 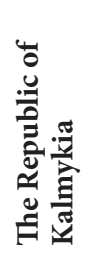 & 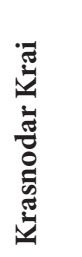 & 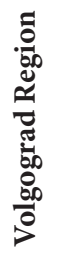 & 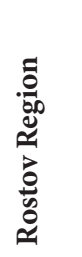 & 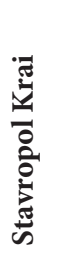 & 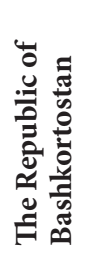 & 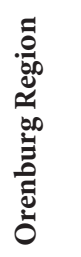 & 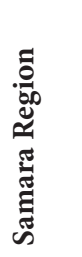 & 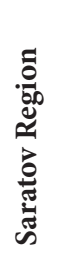 & 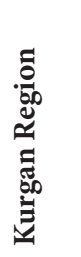 & 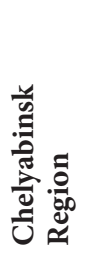 & 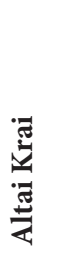 & 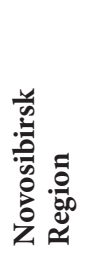 & 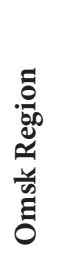 & 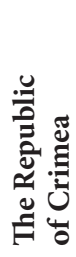 \\
\hline $\mathrm{x}_{5}$ & 29.4 & 29.5 & 16.1 & 39.0 & 32.3 & 36.7 & 29.7 & 26.1 & 28.8 & 36.5 & 26.3 & 34.1 & 38.5 & 33.7 & 31.6 & 32.1 & 25.0 & 44.2 \\
\hline$x_{6}$ & 166.1 & 94.1 & 48.2 & 97.4 & 67.7 & 51.2 & 60.7 & 70.1 & 38.9 & 55.0 & 28.0 & 52.9 & 47.3 & 34.4 & 56.5 & 28.7 & 48.4 & 22.9 \\
\hline $\mathrm{x}_{7}$ & 785.0 & 726.0 & 412.0 & 330.0 & 785.0 & 243.0 & 557.0 & 331.0 & 564.0 & 463.0 & 559.0 & 496.0 & 276.0 & 437.0 & 336.0 & 623.0 & 268.0 & 405.0 \\
\hline $\mathrm{x}_{8}$ & 9.2 & 9.2 & 9.9 & 11.1 & 11.5 & 9.4 & 9.7 & 11.0 & 11.6 & 11.0 & 10.4 & 9.1 & 10.3 & 10.8 & 10.0 & 11.7 & 10.9 & 10.6 \\
\hline $\mathrm{x}_{9}$ & 13.5 & 14.7 & 12.3 & 9.7 & 12.0 & 13.3 & 13.5 & 11.5 & 12.4 & 13.3 & 13.5 & 13.9 & 15.5 & 13.2 & 14.2 & 13.0 & 12.9 & 14.1 \\
\hline $\mathrm{x}_{10}$ & 5.1 & 4.6 & 3.9 & 6.2 & 4.0 & 5.1 & 5.1 & 6.7 & 5.1 & 5.4 & 4.5 & 4.7 & 6.7 & 5.7 & 7.4 & 5.1 & 6.7 & 3.9 \\
\hline $\mathrm{x}_{11}$ & 27.0 & 29.0 & 54.0 & -116.0 & 85.0 & -15.0 & -6.0 & -14.0 & -22.0 & -52.0 & -1.0 & -42.0 & -77.0 & -26.0 & -32.0 & 29.0 & -62.0 & 25.0 \\
\hline $\mathrm{x}_{12}$ & 42.3 & 39.2 & 49.9 & 50.6 & 49.0 & 40.5 & 42.6 & 47.0 & 55.2 & 53.0 & 48.1 & 39.7 & 50.7 & 62.4 & 40.1 & 47.3 & 47.2 & 35.8 \\
\hline $\mathrm{x}_{13}$ & 103.1 & 96.7 & 115.9 & 124.7 & 118.0 & 101.6 & 101.3 & 104.4 & 118.0 & 117.8 & 104.1 & 99.0 & 119.6 & 114.4 & 115.9 & 116.4 & 114.3 & 108.8 \\
\hline $\mathrm{x}_{14}$ & 709.9 & 526.6 & 665.4 & 685.6 & 688.4 & 690.1 & 817.1 & 613.4 & 843.9 & 841.5 & 897.1 & 733.5 & 938.6 & 874.2 & 1089 & 783.9 & 757.1 & 524.9 \\
\hline $\mathrm{x}_{15}$ & 72.2 & 82.1 & 75.2 & 78.3 & 73.5 & 87.7 & 80.3 & 83.7 & 77.4 & 80.7 & 73.9 & 100.8 & 86.7 & 74.2 & 92.0 & 94.8 & 80.7 & 76.3 \\
\hline $\mathrm{x}_{16}$ & 40.5 & 52.3 & 38.9 & 51.8 & 43.4 & 45.1 & 37.5 & 43.9 & 42.8 & 46.7 & 47.7 & 50.5 & 29.7 & 42.0 & 42.7 & 54.0 & 50.8 & 45.7 \\
\hline $\mathrm{x}_{17}$ & 3.6 & 4.4 & 3.5 & 9.2 & 2.5 & 3.8 & 4.0 & 3.4 & 4.8 & 7.0 & 4.5 & 3.9 & 7.1 & 6.2 & 7.5 & 4.2 & 6.2 & 2.6 \\
\hline $\mathrm{x}_{18}$ & 4.8 & 8.1 & 4.3 & 4.7 & 7.4 & 8.8 & 8.5 & 6.7 & 7.8 & 7.6 & 7.6 & 6.8 & 10.3 & 11.5 & 8.8 & 10.4 & 8.4 & 7.0 \\
\hline $\mathrm{x}_{19}$ & 113.1 & 44.7 & 17.6 & 14.7 & 146.1 & 57.8 & 13.6 & 34.0 & 112.3 & 258.8 & 68.5 & 48.3 & 46.7 & 140.4 & 82.3 & 45.1 & 103.9 & 12.0 \\
\hline$x_{20}$ & 5.8 & 2.7 & 1.0 & 0.9 & 2.1 & 3.5 & 1.6 & 1.8 & 6.0 & 3.6 & 4.0 & 2.0 & 1.7 & 5.5 & 1.1 & 1.1 & 4.5 & 1.6 \\
\hline$x_{21}$ & 52.3 & 49.4 & 29.9 & 3.8 & 48.8 & 28.1 & 46.0 & 47.4 & 20.7 & 34.3 & 39.1 & 39.3 & 18.7 & 21.7 & 30.6 & 12.5 & 20.8 & 28.8 \\
\hline$x_{22}$ & 12.9 & 8.5 & 6.2 & 0.8 & 8.3 & 2.9 & 4.5 & 3.8 & 4.7 & 3.3 & 6.7 & 3.6 & 7.9 & 4.6 & 2.3 & 1.5 & 1.7 & 7.2 \\
\hline$x_{23}$ & 1.4 & 1.3 & 2.1 & 0.2 & 2.0 & 6.5 & 1.0 & 0.8 & 0.8 & 2.0 & 1.3 & 2.2 & 0.8 & 3.0 & 0.8 & 0.7 & 0.4 & 2.7 \\
\hline $\mathrm{x}_{24}$ & 8.7 & 8.2 & 36.7 & 0.2 & 20.2 & 4.2 & 2.4 & 1.6 & 39.9 & 4.6 & 12.8 & 6.3 & 22.4 & 29.5 & 22.9 & 27.3 & 32.3 & 8.9 \\
\hline $\mathrm{x}_{25}$ & 77.9 & 57.8 & 44.9 & 7.5 & 48.4 & 46.2 & 50.5 & 49.9 & 43.0 & 71.2 & 53.7 & 68.5 & 57.0 & 82.0 & 75.7 & 87.2 & 93.2 & 55.7 \\
\hline
\end{tabular}


Table 3. The normalized indicators for calculating the EES [EES] index divided into three groups

\begin{tabular}{|c|c|c|c|c|c|c|c|c|c|c|c|c|c|c|c|c|c|c|}
\hline 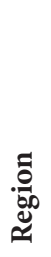 & 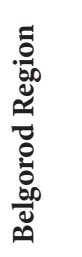 & 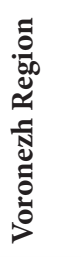 & 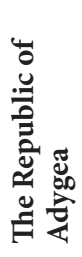 & 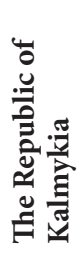 & 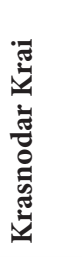 & 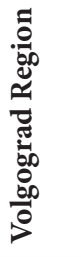 & 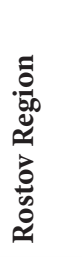 & 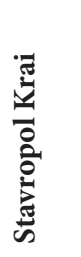 & 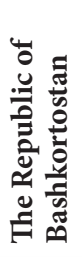 & 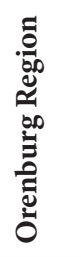 & 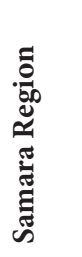 & 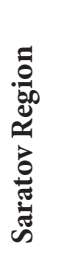 & 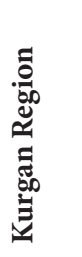 & 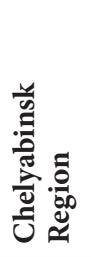 & 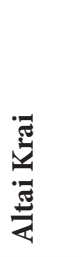 & 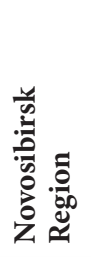 & 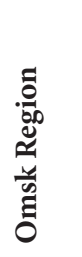 & 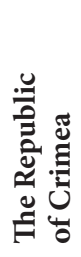 \\
\hline $\mathrm{x}_{1}$ & 1 & 0.409 & 0.209 & 0 & 0.362 & 0.706 & 0.453 & 0.231 & 0.725 & 0.852 & 0.882 & 0.379 & 0.260 & 0.861 & 0.269 & 0.422 & 0.913 & 0.117 \\
\hline $\mathrm{x}_{2}$ & 0.842 & 0.962 & 0.797 & 0 & 1 & 0.466 & 0.852 & 0.632 & 0.840 & .474 & 0.759 & 0.411 & 0.353 & 0.429 & 0.439 & 0.616 & 0.553 & 0.339 \\
\hline$x_{3}$ & 0.454 & 0.476 & 0.117 & 0 & 1 & 0.478 & 0.468 & 0.497 & 0.602 & 0.333 & 0.470 & 0.261 & 0.221 & 0.361 & 0.266 & 0.507 & 0.416 & 0.334 \\
\hline $\mathrm{x}_{4}$ & 0.444 & 0.715 & 0.304 & 0.123 & 0.435 & 0.336 & 0.227 & 0.198 & 0.276 & 0.583 & 0.400 & 0.254 & 0 & 0.344 & 0.120 & 0.310 & 0.233 & 1 \\
\hline $\mathrm{x}_{5}$ & 0.527 & 0.523 & 1 & 0.185 & 0.423 & 0.267 & 0.516 & 0.644 & 0.548 & 0.274 & 0.637 & 0.359 & 0.203 & 0.374 & 0.448 & 0.431 & 0.683 & 0 \\
\hline$x_{6}$ & 1 & 0.497 & 0.176 & 0.520 & 0.313 & 0.197 & 0.264 & 0.329 & 0.111 & 0.224 & 0.035 & 0.209 & 0.170 & 0.080 & 0.235 & 0.040 & 0.178 & 0 \\
\hline $\mathrm{x}_{7}$ & 1 & 0.891 & 0.312 & 0.161 & 1 & 0 & 0.579 & 0.162 & 0.592 & 0.406 & 0.583 & 0.467 & 0.061 & 0.358 & .172 & 0.701 & .046 & 0.299 \\
\hline $\mathrm{x}_{8}$ & 0.038 & 0.038 & 0.308 & 0.769 & 0.923 & 0.115 & 0.231 & 0.731 & 0.962 & 0.731 & 0.500 & 0 & 0.462 & 0.654 & 0.346 & 1 & 0.692 & 0.577 \\
\hline $\mathrm{x}_{9}$ & 0.345 & 0.138 & 0.552 & 1 & 0.603 & 0.379 & 0.345 & 0.690 & 0.534 & 0.379 & 0.345 & 0.276 & 0 & 0.397 & 0.224 & 0.431 & 0.448 & 0.241 \\
\hline $\mathrm{x}_{10}$ & 0.657 & 0.800 & 1 & 0.343 & 0.971 & 0.657 & 0.657 & 0.200 & 0.657 & 0.571 & 0.829 & 0.771 & 0.200 & 0.486 & 0 & 0.657 & 0.200 & 1 \\
\hline $\mathrm{x}_{11}$ & 0.711 & 1.721 & 0.846 & 0 & 1 & 0.502 & 0.547 & 0.507 & 0.468 & 0.318 & 0.572 & 0.368 & 0.194 & 0.448 & 0.418 & 0.721 & 0.269 & 0.701 \\
\hline $\mathrm{x}_{12}$ & 0.246 & 0.128 & 0.531 & 0.558 & 0.495 & 0.177 & 0.256 & 0.423 & 0.729 & 0.648 & 0.462 & 0.149 & 0.560 & 1 & 0.163 & 0.432 & 0.428 & 0 \\
\hline $\mathrm{x}_{13}$ & 0.230 & 0 & 0.684 & 1 & 0.761 & 0.174 & 0.162 & 0.276 & 0.762 & 0.754 & 0.265 & 0.083 & 0.816 & 0.630 & 0.686 & 0.704 & 0.629 & 0.432 \\
\hline $\mathrm{x}_{14}$ & 0.672 & 0.997 & 0.751 & 0.751 & 0.710 & 0.707 & 0.482 & 0.843 & 0.435 & 0.439 & 0.340 & 0.630 & 0.267 & 0.381 & 0 & 0.541 & 0.589 & 1 \\
\hline $\mathrm{x}_{15}$ & 0 & 0.346 & 0.105 & 0.213 & 0.045 & 0.542 & 0.283 & 0.402 & 0.182 & 0.297 & 0.059 & 1 & 0.507 & 0.070 & 0.692 & 0.790 & 0.297 & 0.143 \\
\hline $\mathrm{x}_{16}$ & 0.444 & 0.930 & 0.379 & 0.909 & 0.564 & 0.634 & 0.321 & 0.584 & 0.539 & 0.700 & 0.741 & 0.856 & 0 & 0.506 & 0.535 & 1 & 0.868 & 0.658 \\
\hline $\mathrm{x}_{17}$ & 0.836 & 0.721 & 0.851 & 0 & 0 & 0.811 & 0.783 & 0.875 & 0.653 & 0.330 & 0.700 & 0.801 & 0.317 & 0.450 & 0.258 & 0.745 & 0.444 & 0.987 \\
\hline $\mathrm{x}_{18}$ & 0.938 & 0.475 & 1 & 0.952 & 0.573 & 0.374 & 0.421 & 0.674 & 0.516 & 0.546 & 0.539 & 0.661 & 0.162 & 0 & 0.372 & 0.149 & 0.437 & 0.634 \\
\hline $\mathrm{X}_{19}$ & 0.590 & 0.868 & 0.977 & 0.989 & 0.457 & 0.814 & 0.994 & 0.911 & 0.594 & 0 & 0.771 & 0.853 & 0.859 & 0.480 & 0.715 & 0.866 & 0.628 & 1 \\
\hline
\end{tabular}




\begin{tabular}{|c|c|c|c|c|c|c|c|c|c|c|c|c|c|c|c|c|c|c|}
\hline 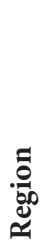 & 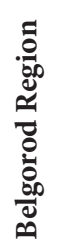 & 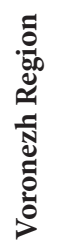 & 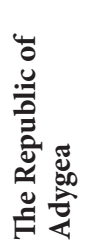 & 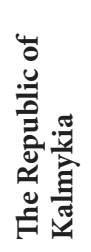 & 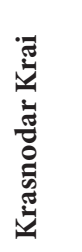 & 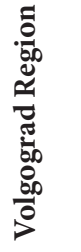 & 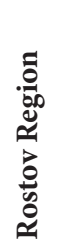 & 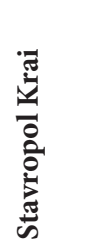 & 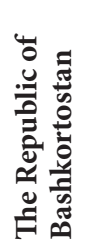 & 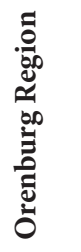 & 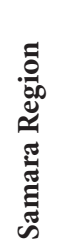 & 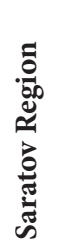 & 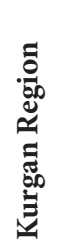 & 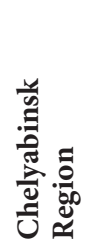 & 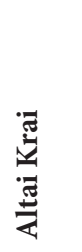 & 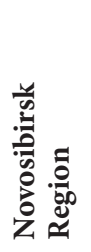 & 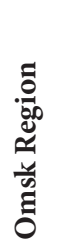 & 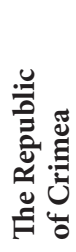 \\
\hline $\mathrm{x}_{20}$ & 0.967 & 0.364 & 0.022 & 0 & 0.248 & 0.520 & 0.134 & 0.178 & 1 & 0.524 & 0.617 & 0.223 & 0.168 & 0.908 & 0.052 & 0.044 & 0.712 & 0.151 \\
\hline $\mathrm{x}_{21}$ & 0 & 0.061 & 0.463 & 1 & 0.072 & 0.500 & 0.131 & 0.101 & 0.653 & 0.371 & 0.272 & 0.268 & 0.693 & 0.632 & 0.449 & 0.821 & 0.650 & 0.485 \\
\hline $\mathrm{x}_{22}$ & 0 & 0.361 & 0.557 & 1 & 0.379 & 0.827 & 0.699 & 0.756 & 0.682 & 0.796 & 0.512 & 0.767 & 0.415 & 0.686 & 0.879 & 0.945 & 0.925 & 0.472 \\
\hline $\mathrm{x}_{23}$ & 0.811 & 0.819 & 0.702 & 1 & 0.717 & 0 & 0.879 & 0.899 & 0.902 & 0.709 & 0.819 & 0.680 & 0.906 & 0.557 & 0.912 & 0.920 & 0.974 & 0.597 \\
\hline $\mathrm{x}_{24}$ & 0.214 & 0.202 & 0.919 & 0 & 0.504 & 0.101 & 0.055 & 0.035 & 1 & 0.111 & 0.317 & 0.154 & 0.559 & 0.738 & 0.572 & 0.683 & 0.809 & 0.219 \\
\hline $\mathrm{x}_{25}$ & 0.821 & 0.587 & 0.436 & 0 & 0.477 & 0.452 & 0.502 & 0.495 & 0.414 & 0.743 & 0.539 & 0.712 & 0.578 & 0.869 & 0.796 & 0.930 & 1 & 0.562 \\
\hline
\end{tabular}




\section{Results}

Analyzing the initial data, we found that in the regions studied, the maximum value of areas with stable landscape components belongs to the Novosibirsk region - 12,489 thousand ha; the Omsk region - 9,259.3 thousand ha; and the Republic of Bashkortostan - 9,884.8 thousand ha. These indicators are due to a large part of the forest, pastures, and hayfields. In the Novosibirsk and Omsk regions, vast areas are occupied by swamps (3,059.6 and 2,026.8 thousand ha, respectively). In the Belgorod region and the Republic of Crimea, pastures and forests predominate; but in the Republic of Adygea, 59.4\% of sustainable landscapes are occupied by forests. The maximum values of the parameters indicating the instability of the landscape components were observed in Altai Krai - 7,058.3 thousand ha, Rostov region 6545.3 thousand ha, and Orenburg region - 6,535.1 thousand ha. Villages occupy more than $80 \%$ of the total area of unstable landscapes; In the Republic of Crimea, 69.4\% of unstable landscapes are occupied by arable land. Most of the plots under construction are in Krasnodar Krai - 202.9 thousand ha. It represents 4.2\% of the total area of unstable landscapes. Furthermore, the Volgograd and Orenburg regions are characterized by a high indicator of construction plots - 169.5 thousand ha $(2.6 \%)$ and -158.7 thousand ha $(2.4 \%)$, respectively; We note fewer construction plots in the Republic of Adygea (22.1 thousand ha), the Republic of Kalmykia (32.2 thousand ha) and the Kurgan Region - 49.1 thousand ha. Most of the roads are located in the Republic of Bashkortostan - 260.1 thousand ha, the Rostov region - 196 thousand ha; the least roads are found in the Republic of Adygea - 18.8 thousand ha and the Republic of Crimea - 43.4 thousand ha. The maximum area of reclaimed land is located in Krasnodar Krai - 496 thousand ha, the Republic of Crimea - 397.3 thousand ha, and the Rostov and Saratov regions - 259.6 and 257.8 thousand ha, respectively; the smallest number of reclaimed land belongs to the Republic of Adygea -27.3 thousand ha and the Kurgan region - 30.6 thousand ha.

As a result of the assessment of landscape-ecological stability, we found that only the Republic of Kalmykia has pronounced stability of landscapes $(5,8)$, the coefficient of which exceeds the average four times (Table 4). To the greatest extent, landscapes are characterized as unstable (7 regions); we noted the lowest coefficient of stability of the ecological landscape (0.4) in the Belgorod Region, Stavropol $\mathrm{Krai}$, and the Republic of Crimea. In general, stable landscape components dominate over landscapes with unstable components (i.e., where CELS $>0$ ) in economically underdeveloped regions (the Republic of Kalmykia and the Kurgan Region) and relatively large territories (the Novosibirsk and Omsk Regions, the Republic of Bashkortostan, and Altai Krai). The correlation analysis partially confirms the impact of the economic development of the region on the landscape and ecological stability. The relationship between CELS and the Economic Development Index $\left(\mathrm{P}_{\mathrm{I}}\right)$ is reversed; on the Cheddock scale, it is characterized by a significant coupling force $(-0.5)$. We observed a proportional relationship between the CELLS and the indicator of the ecological state of the territory $\left(\mathrm{P}_{\text {III }}\right)$. Therefore, the correlation coefficient is 0.5 and a significant binding force characterizes the dependence. 
The EES analysis shows the minimum dispersion of the values of the integral indicator - of 1.1 to 1.8 on a scale from 1 to 3 . This fact does not allow us to draw an unambiguous conclusion about the disproportion of the existing ecological and economic situation between the studied regions. In this case, it is advisable to consider the differences between the indices in each region separately. We noticed the maximum values of the economic development index (more than 0.7) in the Belgorod region (due to the maximum parameters characterizing the shipped goods of local production, agricultural products, and the commissioning of residential buildings) and in Krasnodar Krai (due to the maximum parameters characterizing the retail trade turnover, the volume of paid services to the population, and the commissioning of residential buildings). Furthermore, this indicator significantly ranges from 0.16 in the Republic of Kalmykia to 0.71 in the Belgorod region. A disproportion in economic development characterizes the regions studied. We found minimal differences between the regions in the social sector (the index values range from 0.32 in the Kurgan region to 0.7 in Krasnodar Krai). This fact indicates a partial leveling of the disparity and a relatively regular distribution of funds from the federal budget for the needs of the social sector in the regions.

Table 4. Integral table of the coefficient of stability of the ecological landscape and the index of EES of the steppe regions of Russia

\begin{tabular}{lllllllll}
\hline No. & Region & $\begin{array}{l}\mathbf{F}_{\text {st' }} \text { thou. ha } \\
\text { thou. ha }\end{array}$ & $\begin{array}{l}\mathbf{F}_{\text {uns' }} \\
\text { thoLS }\end{array}$ & $\mathbf{P}_{\mathrm{I}}$ & $\mathbf{P}_{\text {II }}$ & $\mathbf{P}_{\text {III }}$ & I $^{\text {ees }}$ \\
\hline 1 & Belgorod Region & 756.3 & 1824.9 & 0.4 & 0.71 & 0.47 & 0.49 & 1.7 \\
2 & Voronezh Region & 1588.6 & 3361.4 & 0.5 & 0.68 & 0.48 & 0.47 & 1.6 \\
3 & The Republic of Adygea & 485.8 & 328.1 & 1.5 & 0.45 & 0.64 & 0.58 & 1.7 \\
4 & The Kalmyk Republic & 5757.6 & 986.5 & 5.8 & 0.16 & 0.59 & 0.57 & 1.3 \\
5 & Krasnodar Krai & 2819.3 & 4815.7 & 0.6 & 0.70 & 0.70 & 0.41 & 1.8 \\
6 & Volgograd Region & 3566.5 & 6321.2 & 0.6 & 0.29 & 0.46 & 0.46 & 1.2 \\
7 & Rostov Region & 2965.2 & 6545.3 & 0.5 & 0.48 & 0.41 & 0.48 & 1.4 \\
8 & Stavropol Krai & 1928.4 & 4498.6 & 0.4 & 0.41 & 0.56 & 0.48 & 1.5 \\
9 & The Republic of Bashkortostan & 9884.8 & 4153.1 & 2.4 & 0.49 & 0.59 & 0.74 & 1.8 \\
10 & Orenburg Region & 5436.9 & 6535.1 & 0.8 & 0.38 & 0.52 & 0.46 & 1.4 \\
11 & Samara Region & 1926.7 & 3308.7 & 0.6 & 0.48 & 0.49 & 0.55 & 1.5 \\
12 & Saratov Region & 3226.5 & 6504.0 & 0.5 & 0.33 & 0.51 & 0.52 & 1.4 \\
13 & Kurgan Region & 4208.6 & 2569.7 & 1.6 & 0.17 & 0.32 & 0.60 & 1.1 \\
14 & Chelyabinsk Region & 5000.6 & 3443.4 & 1.5 & 0.32 & 0.46 & 0.70 & 1.5 \\
15 & Altai Krai & 8800.9 & 7058.3 & 1.2 & 0.28 & 0.34 & 0.63 & 1.2 \\
16 & Novosibirsk Region & 12489.0 & 4126.7 & 3.0 & 0.43 & 0.65 & 0.74 & 1.8 \\
17 & Omsk Region & 9259.3 & 4530.7 & 2.0 & 0.35 & 0.48 & 0.81 & 1.7 \\
18 & The Republic of Crimea & 813.2 & 1832.6 & 0.4 & 0.33 & 0.58 & 0.50 & 1.4 \\
\hline & & & & & & & &
\end{tabular}




\section{Discussion}

We designed a generalized schematic map based on the calculated coefficients and integral indicators (Fig. 1). It represents the spatial disproportions of landscapeecological stability and EES of the steppe regions of Russia. We established that the level of ecological sustainability of landscapes increases in the north-eastern regions, except for the Republic of Kalmykia and the Republic of Adygea, characterized by weak development of industry and infrastructure. In regions where CELS is greater than 1 (where landscapes with stable components predominate), the distribution of EES indicators has the proportion of 1/1.5/2.

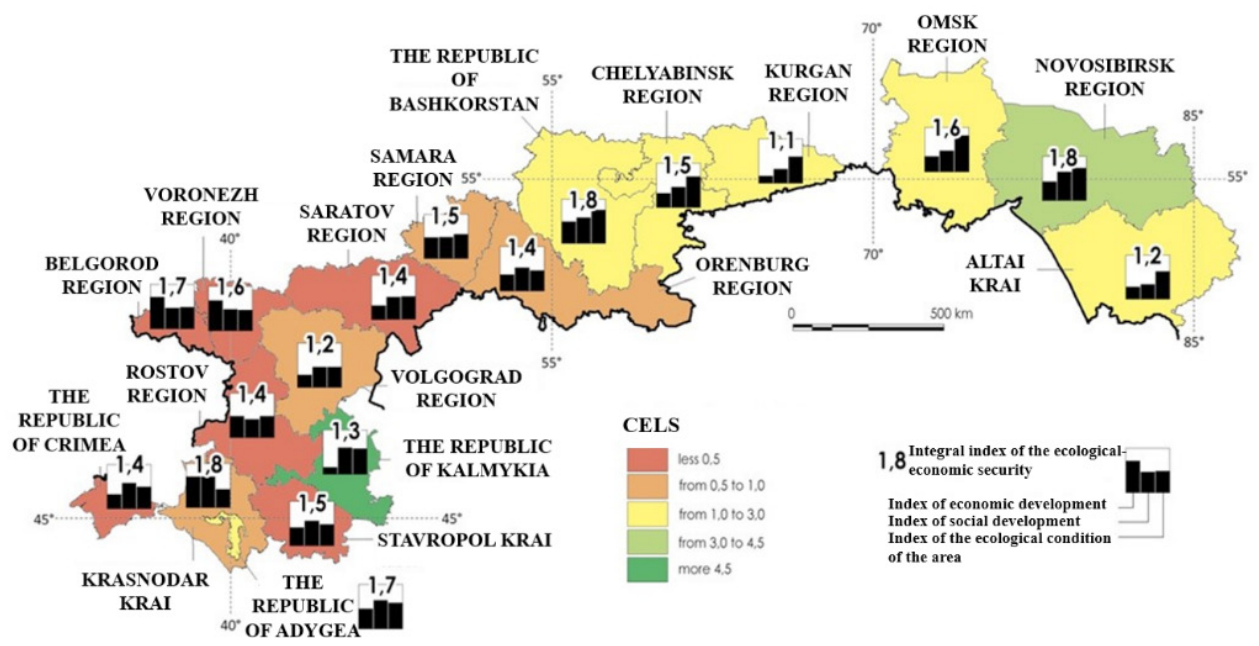

Figure 1. Schematic map of landscape-ecological stability and EES of the regions of the steppe zone of Russia. Source: (Federal State Statistics Service 2018).

Studying CELS and EES integral indexes, one would conditionally define welldeveloped and poorly developed regions in the social-economic sector and the condition of landscape and environment. The Republic of Bashkortostan and the Novosibirsk Region could be considered well-developed regions. In general, their landscapes are characterized by relative stability where the integral index of the EES reaches the maximum value (1.8). The Volgograd region could be regarded as a poorly developed region characterized by a low level of ecological-economic security and unstable landscapes.

\section{Discussion}

A high level of agricultural development characterizes the steppe regions of the southern European part of Russia. Large-scale water and chemical reclamation in these territories aggravate the conditions of soil resources. One of the main envi- 
ronmental problems is the restoration of the biopotential of soil resources in the western part of the study region. To change this situation for the better, one should use soil protection and cultivation of arable land, new irrigation technology, soil reclamation, forest reclamation, pasture restoration, and many other measures.

Despite the relatively high value of CELS in the Republic of Kalmykia, this region is characterized by increased soil degradation due to desertification, erosion, soil salinization, and land loading.

The ecological problems of the Volga and the southern Ural basin are characterized by the destruction of ecosystem connections and the threat to human health. A difficult hydrochemical situation has developed in the water bodies of the Kuibyshev and Saratov reservoirs. The reduction in annual flow of the Volga and Ural rivers is aggravated by the loss of a unique capability - natural self-purification and the destruction of floodplain and fish potential.

As a result of economic activity in the central part of the studied mesoregion, industrial waste is generated, including the products of processing enterprises. Landfills occupy a thousand hectares of land.

Almost all major industrial centers of the Urals and Siberia aggravate the ecological situation outside the studied mesoregion.

Therefore, the spatial analysis of the landscape-ecological stability and ecologicaleconomic security of the steppe regions of Russia and its assessment revealed many urgent environmental problems.

In the southern European part of the steppe regions of Russia, we identified such problems as (1) soil disruption due to oil and gas production, (2) secondary salinization and soil deflation, (3) violation of the regime of specially protected areas, (4) deterioration of natural meadows and (5) atmospheric air pollution.

In the central part of the steppe zone of Russia, we encounter (1) soil disruption as a result of oil and gas production and mining, (2) soil erosion, (3) loss of productive land, (4) ravine formation, (5) depletion and pollution of water resources, (6) deforestation, (7) degradation of forests and (8) air pollution.

In the eastern part of the steppe zone of Russia, we determined the following issues: (1) loss of productive land, (2) depletion and pollution of water resources, (3) exhaustion of fish resources and commercial fauna, (4) soil deflation, and (5) air pollution.

\section{Acknowledgements}

The paper is done within the framework of the project Steppes of Russia: landscape-ecological bases of stable development, the basics of nature-like technologies in the conditions of natural and anthropogenic changes in the environment (No. GRAAAA-A17-117012610022-5). 


\section{References}

Baranov VA (1995) Ecological, Sustainable, and Optimal Development of Agricultural Landscapes. In: Popov AI (Ed.) The program of transition of the Saratov region to the model of sustainable development. Saratov State Technical University, Saratov, 112-114. [In Russian]

Baranov VA (2001) Environmental Sustainability and Optimization of Agricultural Landscapes and Agroecosystems. Russian State Agrarian Extramural University, Moscow. [In Russian]

Baranov VA (2012) Optimization of Agroforestry Landscapes in the South-East of European Russia. ASP, Saratov, 274 pp. [In Russian]

Carpenter SR, Brock WA, Hanson P (1999) Ecological and Social Dynamics in Simple Models of Ecosystem Management. Conservation Ecology 3 (2): 10. https:// doi.org/10.5751/ES-00122-030204

Chapin FS, Carpenter SR, Kofinas GP, Folke C, Abel N, Olsson P, Swanson FJ (2010) Ecosystem Stewardship: Sustainability Strategies for a Rapidly Changing Planet. Trends in Ecology \& Evolution 25 (4): 241-249. http://dx.doi.org/10.1016/j. tree.2009.10.008

Federal Service for State Registration, Cadastral Records, and Cartography: State report on the status and use of land in the Russian Federation. Rosreestr, Moscow. https://rosreestr.ru/site/activity/sostoyanie-zemel-rossii/gosudarstvennyy-natsionalnyy-doklad-o-sostoyanii-i-ispolzovanii-zemel-v-rossiyskoy-federatsii/ [In Russian]

Federal State Statistics Service: Regions of Russia. Socio-economic indicators. Rosstat, Moscow. https://gks.ru/bgd/regl/b19_14p/Main.htm [In Russian]

Glinsky VV, Serga LK, Khvan MS (2015) Environmental Safety Assessment of Municipalities in the Region: Scorecard, Estimation Method, and its Application. Ideas and Ideals 4 (26): 13-32. [In Russian]

Holling CS (Ed) (1978) Adaptive Environmental Assessment and Management. John Wiley \& Sons, New York.

Jongman RHG, Külvik M, Kristiansen I (2004) European Ecological Networks and Greenways. Landscape and Urban Planning 68 (2-3): 305-319. http://dx.doi.or g/10.1016\%2FS0169-2046(03)00163-4

Klementova EN, Heinige V (1995) Assessment of the Ecological Sustainability of the Agricultural Landscape. Melioration and Water Management 5: 33-34. [In Russian]

Klyushin PV, Shormanov AK (2015) Generalized Assessment of Anthropogenic Load in the Kabardino-Balkarian Republic. State University of Land Use Planning, Moscow. [In Russian]

Kochurov BI (2005) Modern Land Management and Management of Land Use in Russia. In: Glazovsky NF (Ed.) Stable agriculture and rural development: Foreign experience and problems of Russia. KMK, Moscow, 322-334. [In Russian] 
Malezieux E (2012) Designing Cropping Systems from Nature. Agronomy for Sustainable Development 32 (1): 15-29.

McNeely J, Scherr S (2003) Ecoagriculture: Strategies to Feed the World and Save Wild Biodiversity. Island Press, Washington, Covelo, London.

Rusak ON (2011) Current Problems of Ecological Security. Kompetentnost 6: 4446. [In Russian]

Saprin SV (2017) Assessment of ecological sustainability of agricultural landscapes of the Voronezh Region. Dissertation of Candidate of Geographical Sciences. Voronezh State Agrarian University named after Emperor Peter the Great, Voronezh, Russia. [In Russian]

Stinner BR, Lorenzoni GG, Maurizio P (Ed.) (1989) Agricultural Ecology and Environment. Elsevier, Padova.

Tronin AA (2013) Remote Sensing in Ecological Safety. Current Problems in Remote Sensing of The Earth from Space 10 (1): 238-245. [In Russian]

Vlasova EY (2008) Strategic Directions of Ensuring Environmental Security of the Region. Fundamental Research 5: 194-197. [In Russian]

Volovich VN (2012) On the Issue of Environmental Security of the Country. Society. Environment. Development (Terra Humana) 1: 192-196. [In Russian]

Zhaoxue LI, Linyu X (2010) Evaluation Indicators for Urban Ecological Security Based on Ecological Network Analysis. Procedia Environmental Sciences 2: 1393-1399. http://dx.doi.org/10.1016/j.proenv.2010.10.151 